\title{
Calidad y satisfacción del paciente en el manejo laparoscópico ambulatorio de patología vesicular no grave y no complicada
}

\author{
Quality and patient satisfaction after ambulatory laparoscopic management of non-severe \\ and uncomplicated gallbladder pathology
}

Daniel Holguín-Ruacho y Carlos R. Cervantes-Sánchez ${ }^{2 *}$

${ }^{1}$ Servicio de Cirugía General; ²Subdirección de Enseñanza e Investigación. Hospital General Dr. Salvador Zubirán Anchondo, Chihuahua, Chihuahua, México

\begin{abstract}
Resumen
Introducción: La colecistectomía laparoscópica ambulatoria (CLA) disminuye la utilización de camas y beneficia al paciente al no hospitalizarse, siendo importante evaluar su grado de satisfacción. Material y métodos: Se diseñó un ensayo clínico controlado para eficacia, seguridad y satisfacción de CLA comparándola con colecistectomía laparoscópica habitual (CLH). Eficacia y seguridad se evaluaron en cuanto; recuperación de vía oral, micción espontánea, canalización de gases, dolor, nausea, vómito, sangrado, y gasto del penrose. La satisfacción a través de una adaptación del cuestionario SATISCORE. Resultados: Se incluyeron 71 pacientes, 38 del grupo experimental (CLA) y 33 del control (CLH). En ambos hubo una mayor proporción de mujeres, edad promedio en la quinta década, y distribución de comorbilidades semejante. En seguridad y eficacia, los resultados fueron semejantes. En la encuesta el grupo de CLA manifestó $89.5 \%$ de alto grado de satisfacción. Discusión: Hubo una buena recuperación en ambos grupos, con una reincorporaron más temprana y un mejor índice de satisfacción referido por el enfermo en el grupo experimental (CLA). Conclusión: La CLA es tan efectiva y segura como la CLH, por lo que se puede considerar como el nuevo "Estándar de oro" para el manejo quirúrgico de la patología biliar no complicada.
\end{abstract}

PALABRAS CLAVE: Colecistectomía laparoscópica. Colelitiasis. Ensayo clínico controlado. Humanos. Patología vesicular. Procedimientos ambulatorios.

\begin{abstract}
Introduction: Ambulatory laparoscopic cholecystectomy $(A L C)$ reduces the use of hospital beds and benefits the patient by returning home sooner. Therefore, it is important to evaluate the degree of patient satisfaction. Materials and methods: $A$ controlled clinical trial was designed to assess the efficacy, safety, and satisfaction of ALC, compared to the usual laparoscopic cholecystectomy (ULC). Efficacy and safety were evaluated based on; recovery of the oral route, spontaneous urination, gas channeling, presence of pain, nausea, vomiting, bleeding, pain, penrose expense. Satisfaction was explored through an adaptation of the SATISCORE questionnaire. Results: We included 71 patients, 38 from the experimental group (ALC) and 33 from the control group (ULC). There was a higher proportion of women, average age at the fifth decade, and a similar distribution of comorbidities. In safety and efficacy, the results were similar. In the survey, the ALC group showed an 89.5\% high degree of satisfaction. Discussion: There was a good postoperative recovery in both groups as they reincorporated earlier, identified in a better satisfaction index referred by the patient. Conclusion: The ALC is as effective and safe as ULC, so it can be considered as the new "gold standard" for the surgical management of uncomplicated biliary pathology.
\end{abstract}

KEY WORDS: Laparoscopic cholecystectomy. Cholelithiasis. Controlled clinical trial. Humans. Gallbladder pathology. Ambulatory surgical procedures.

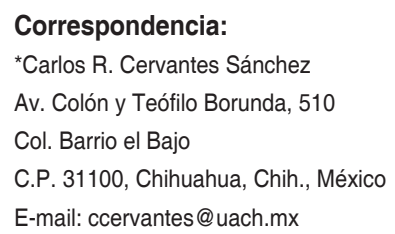

Fecha de recepción: 23-10-2018

Fecha de aceptación: 25-02-2019 DOI: $10.24875 / C I R U .19000833$
Cir Cir. 2019;87:656-661 www.cirugiaycirujanos.com 


\section{Introducción}

La primera descripción de patología vesicular de origen litiásico data del siglo vi y se adjudica a Alejandro de Tralles, mientras que el manejo quirúrgico surge a finales del siglo xvi, y después aparecen la antisepsia (1846) y la anestesia (1867). La primera colecistectomía abierta fue realizada por Langenbuch en 1882, y gracias a los avances técnicos y científicos comienza en 1985 la era de la cirugía laparoscópica con Mühe en Alemania, hasta posicionarse el día de hoy como la técnica de elección'.

Numerosos estudios muestran los beneficios del empleo de esta técnica, tanto para el paciente como para el sistema de salud, al reducir los días de estancia hospitalaria y acortar los periodos de convalecencia de quien requiere la cirugía ${ }^{2}$. Las ventajas clínicas posoperatorias de este tipo de procedimiento incluyen disminución del dolor, menor limitación respiratoria y menor frecuencia de íleo, y por lo tanto una mejor evolución hacia la recuperación ${ }^{3}$.

Debido a los beneficios y las ventajas clínicas señaladas, la tendencia actual es a incorporar procedimientos mayores, como la colecistectomía laparoscópica, en los programas de cirugía ambulatoria, la cual se considera como tal cuando es un evento programado a priori de manera electiva en el que la admisión, la realización del procedimiento, la recuperación y el alta suceden el mismo día, por lo que se requiere una adecuada selección de los $\operatorname{casos}^{4}$. Sin embargo, son factores limitantes para la aceptación de esta técnica el temor al dolor, las náuseas o vómitos posoperatorios, y la edad avanzada ${ }^{5}$.

La colecistectomía laparoscópica (CL) es el método de referencia para el manejo de la colecistopatía no complicada. Es un procedimiento electivo en el cual la admisión y la realización ocurren el mismo día, con inicio de la recuperación al término de la cirugía, y habitualmente el alta sucede al día siguiente, con la intención de identificar la ocurrencia de molestias o complicaciones, así como de evaluar la recuperación de la funcionalidad del tracto digestivo, que se altera por el procedimiento anestésico.

Con la implementación de la CL ambulatoria (CLA) puede aumentarse la actividad quirúrgica sin estar limitados por la disponibilidad de camas censables, con un impacto positivo en el aspecto económico para las instituciones de salud, sin dejar de mencionar el potencial beneficio para el paciente al regresar el mismo día a la comodidad de su hogar. Por lo tanto, en un hospital que cuente con el personal capacitado y la tecnología para la realización de este procedimiento, sin sacrificar la calidad y la seguridad, será importante evaluar el grado de satisfacción del paciente.

\section{Método}

Se diseñó un ensayo clínico controlado para conocer la eficacia, la seguridad y la satisfacción de la CLA comparándola con la CL habitual (CLH), en el servicio de cirugía del Hospital General de Chihuahua Dr. Salvador Zubirán Anchondo, en pacientes procedentes de la consulta externa con diagnóstico de colelitiasis o colecistitis no complicadas, entre junio y diciembre de 2017.

La eficacia y la seguridad se evaluaron en función de la recuperación de la vía oral, micción espontánea, canalización de gases o evacuación rectal, presencia de dolor, náuseas o vómitos, complicaciones inmediatas como sangrado o dolor, y las características del gasto por el Penrose.

La satisfacción se midió con una adaptación del cuestionario SATISCORE, originalmente diseñado para cirugía cardiaca ${ }^{6}$. Dicha adaptación se conformó por 10 preguntas con respuestas bajo una escala Likert de cuatro niveles (nada satisfecho, poco satisfecho, satisfecho o muy satisfecho), en la que si se obtienen entre 10 y 15 puntos se considera un alto grado de satisfacción, de 16 a 25 puntos se considera un grado de satisfacción moderada, y una puntuación mayor de 26 se considera insatisfacción con la atención recibida (Anexo 1).

El tamaño de muestra se calculó con la fórmula para estimar una proporción con un nivel de confianza del 95\%, una proporción esperada del $97 \%$ y una precisión del $5 \%$, resultando en 66 casos tras considerar una probabilidad de un $10 \%$ de pérdidas durante el seguimiento.

La asignación de los pacientes a cada grupo fue por muestreo aleatorio mediante un sistema electrónico en línea, incluyendo pacientes del seguro popular y del Instituto Mexicano del Seguro Social que fueron programados en nuestro hospital mediante un convenio de colaboración interinstitucional estatal.

Las cirugías se programaron de forma convencional para ambos grupos, con previa realización de estudios y valoraciones preoperatorias de forma habitual, cuidando programarlas en el primer turno del día, es decir, a las 8:00 horas. 
Los criterios de inclusión fueron ambos sexos, edad entre 18 y 74 años, riesgo quirúrgico ASA (American Society of Anesthesiologists) I, II y III, enfermedades degenerativas crónicas (diabetes, hipertensión, síndrome metabólico u obesidad) estables y con adecuado control en el momento de la programación, residentes en la ciudad de Chihuahua (o que contaran con facilidades para su traslado en caso de ser necesario) y que aceptaran participar en el estudio mediante un consentimiento informado escrito, previamente validado y aceptado por el Comité de Ética en Investigación del Hospital (debidamente registrado en la Conbioética; CONBIOÉTICA-08-CEI-001-20170517) después de haber sido revisado, aprobado y registrado el protocolo de estudio, el cual se apegó a los lineamientos del Reglamento de la Ley General de Salud en Materia de Investigación para la Salud ${ }^{7}$ y a la Declaración de Helsinki de 1964 y su última enmienda en Fortaleza, Brasil $(2013)^{8}$ en apego a las buenas prácticas de investigación clínica.

Los criterios de exclusión fueron presencia de vesícula escleroatrófica en la ultrasonografía, enfermedad litiásica complicada o grave (colecistitis agudizada, piocolecisto, coledocolitiasis, pancreatitis aguda o carcinoma vesicular), índice de masa corporal mayor de 35 , cirrosis hepática, enfermedad pulmonar obstructiva crónica, cardiopatía y embarazo.

Los criterios de eliminación incluyeron tiempo quirúrgico mayor de 2 horas, conversión a cirugía abierta y pacientes ambulatorios en los que, por alguna razón médica, se tomara la decisión de hospitalizar, registrando dicha razón.

\section{Resultados}

Se incluyeron 71 pacientes programados para CL que cumplieron con los criterios de inclusión, de los que 38 fueron asignados al grupo experimental (CLA) y 33 al grupo control (CLH). En ambos grupos se observó una mayor proporción de mujeres: 84.2 y $90.9 \%$, respectivamente. Las edades promedio fueron de $41.02 \pm 13.43$ y $42.24 \pm 13.95$, respectivamente. La distribución de la comorbilidad (diabetes, hipertensión, síndrome metabólico u obesidad) fue muy semejante entre ambos grupos (Tabla 1).

Con respecto a la tolerancia a la vía oral, en el grupo de CLA el $84 \%$ la recuperó en la evaluación que se realizó en la tarde del mismo día de la cirugía (a las 19:00 h), mientras que lo hizo el $72 \%$ del
Tabla 1. Características demográficas

\begin{tabular}{lcc}
\hline & $\begin{array}{c}\text { Colecistectomía } \\
\text { laparoscópica } \\
\text { habitual }\end{array}$ & $\begin{array}{c}\text { Colecistectomía } \\
\text { laparoscópica } \\
\text { ambulatoria }\end{array}$ \\
\hline $\begin{array}{l}\text { Sexo } \\
\text { Masculino }\end{array}$ & $3(9.1 \%)$ & $6(15.8 \%)$ \\
Femenino & $30(90.9 \%)$ & $32(84.2 \%)$ \\
Diabetes mellitus & $42.24 \pm 13.9$ & $41.02 \pm 13.4$ \\
Hipertensión & $2(6.06 \%)$ & $1(2.63 \%)$ \\
Síndrome metabólico & $2(6.06 \%)$ & $8(21.05 \%)$ \\
Obesidad & $0(0 \%)$ & $4(10.52 \%)$ \\
Total & $1(3.03 \%)$ & $2(5.26 \%)$ \\
\hline
\end{tabular}

grupo de CLH, sin alcanzar una diferencia estadísticamente significativa $(p=0.2404)$, ni al día siguiente $(p=0.3513)$. Tampoco se encontró diferencia significativa en cuanto a la presencia de náuseas la tarde del mismo día $(p=0.7114)$, ni al día siguiente $(p=0.9198)$. El vómito se presentó en cuatro casos del grupo de CLA y en cinco casos del grupo de CLH en la tarde del mismo día ( $p=0.8207$ ), mientras que al día siguiente se presentó solo en un caso del grupo de CLA ( $p=0.3513)$. El dolor referido por ambos grupos también fue semejante tanto la tarde del mismo día ( $p=0.5185)$ como al día siguiente $(p=0.9826)$. La recuperación de la diuresis espontánea en el posoperatorio inmediato también fue semejante en ambos grupos $(p=1)$. El $31.6 \%$ de los casos del grupo de CLA refirieron canalizar gases por el recto la misma tarde del procedimiento, llegando al $92.1 \%$ al día siguiente, mientras que lo hicieron el $42.4 \%$ del grupo de CLH la misma tarde y el $90.9 \%$ al día siguiente $(p=0.3475$ y $p=0.8575$, respectivamente) (Tabla 2 ).

El sangrado transoperatorio fue mínimo (menor de $50 \mathrm{~cm}^{3}$ ) en todos los pacientes de ambos grupos, sin presentar inestabilidad hemodinámica ni necesidad de transfusión ( $p=1)$, mientras que el uso del drenaje mostró una diferencia significativa $(p=0.0002)$ al haber sido empleado solo en dos casos del grupo de CLA y en 14 del grupo de CLH, en relación con las preferencias del médico tratante (Tabla 3).

En cuanto a la encuesta aplicada a todos los pacientes, se observaron mejores grados de satisfacción en los del grupo de CLA: 34 pacientes (89.5\%) reportaron un alto grado de satisfacción y 4 (10.5\%) una satisfacción media-moderada, mientras que en el grupo de CLH $27(81.8 \%)$ refirieron un alto grado 
Tabla 2. Recuperación posoperatoria (eficacia)

\begin{tabular}{|c|c|c|c|}
\hline & $\begin{array}{l}\text { Colecistectomía } \\
\text { laparoscópica habitual }\end{array}$ & $\begin{array}{c}\text { Colecistectomía laparoscópica } \\
\text { ambulatoria }\end{array}$ & Prueba de hipótesis y $p$ \\
\hline \multicolumn{4}{|l|}{ Tolerancia oral } \\
\hline Día 0 & $24(72.72 \%)$ & $32(84.21 \%)$ & $X^{2}=1.37(p=0.24)$ \\
\hline Día 1 & $33(100 \%)$ & $37(97.36 \%)$ & $X^{2}=0.86(p=0.35)$ \\
\hline \multicolumn{4}{|l|}{ Náuseas } \\
\hline Día 0 & $10(30.30 \%)$ & $10(26.31 \%)$ & $X^{2}=0.13(p=0.71)$ \\
\hline Día 1 & $1(3.03 \%)$ & $1(2.63 \%)$ & $X^{2}=0.01(p=0.91)$ \\
\hline \multicolumn{4}{|l|}{ Vómitos } \\
\hline Día 0 & $5(15.15 \%)$ & $4(10.52 \%)$ & $X^{2}=0.05(p=0.82)$ \\
\hline Día 1 & $0(0 \%)$ & $1(2.63 \%)$ & $X^{2}=0.86(p=0.35)$ \\
\hline \multicolumn{4}{|l|}{ Dolor } \\
\hline \multicolumn{4}{|l|}{ Día 0} \\
\hline Nulo & $0(0 \%)$ & $1(2.63 \%)$ & \\
\hline Leve & $18(54.54 \%)$ & $23(60.52 \%)$ & $X^{2}=2.26(p=0.51)$ \\
\hline Moderado & $14(42.42 \%)$ & $14(36.84 \%)$ & \\
\hline Intenso & $1(3.03 \%)$ & $0(0 \%)$ & \\
\hline \multicolumn{4}{|l|}{ Día 1} \\
\hline Nulo & $4(12.12 \%)$ & $5(13.15 \%)$ & \\
\hline Leve & $27(81.81 \%)$ & $31(81.57 \%)$ & $X^{2}=0.03(p=0.98)$ \\
\hline Moderado & $2(6.06 \%)$ & $2(5.26 \%)$ & \\
\hline Intenso & $0(0 \%)$ & $0(0 \%)$ & \\
\hline \multicolumn{4}{|l|}{ Diuresis } \\
\hline Día 0 & $33(100 \%)$ & $38(100 \%)$ & $X^{2}=1.00(p=1)$ \\
\hline \multicolumn{4}{|l|}{ Gases por recto } \\
\hline Día 0 & $14(42.42 \%)$ & $12(31.57 \%)$ & $X^{2}=0.88(p=0.34)$ \\
\hline Día 1 & $30(90.90 \%)$ & $35(92.10 \%)$ & $X^{2}=0.03(p=0.85)$ \\
\hline
\end{tabular}

de satisfacción y 4 (12.12\%) una satisfacción media-moderada, aunque sin significancia estadística ( $p=0.5929)$ (Tabla 4).

\section{Discusión}

En México se han publicado tres estudios que reportan el uso de CLA para el manejo de la colecistopatía, con buenos resultados. El primero se publicó en 2004 y es un reporte de un hospital de segundo nivel que incluyó 405 CLA realizadas en un periodo de 10 años, de las que 306 (75.5\%) fueron exitosas, sin mortalidad y con una morbilidad del $10.1 \%$, por lo que los autores consideraron que su programa era factible y seguro ${ }^{9}$. En 2008 se publicó un estudio realizado en otro hospital de segundo nivel en el que se realizaron 907 procedimientos de CLA en un periodo de 8 años, de los que ninguno requirió ingreso hospitalario ni atención en urgencias, sin mortalidad y solo con dos pacientes con equimosis y un hematoma periumbilicales, reportando un ahorro de $\$ 3,180.00$ M.N. por paciente ${ }^{2}$. Por último, en 2011 se publicó un estudio realizado en una unidad de cirugía ambulatoria en el que se incluyeron 597 casos de $\mathrm{CL}$, durante un periodo de 2 años, de los que 553 fueron manejados como CLA, sin mortalidad ni morbilidad ${ }^{10}$. Sin embargo, en ninguno de estos estudios se evaluó el grado de satisfacción de los pacientes operados.

En el presente estudio, los dos grupos fueron semejantes en todas las variables consideradas (demográficas, clínicas y quirúrgicas). No hubo conversiones ni necesidad de internamiento o manejo posoperatorio inmediato en el servicio de urgencias. El uso de drenaje se permitió a discreción del médico tratante, pero en ninguno de los dos grupos fue necesario su uso sistemático y no interfirió en el manejo del dolor, la evolución ni las posibles complicaciones. En todos los casos hubo una buena tolerancia a la vía oral, una adecuada remisión del dolor y una similar recuperación del tránsito intestinal, así como un nulo índice de infección posquirúrgica, por lo que los pacientes se reincorporaron de manera más temprana a su vida cotidiana, lo cual se identificó en un mejor índice de satisfacción referido por el enfermo para el caso de la CLA. En general no se muestra ninguna desventaja de la cirugía laparoscópica ambulatoria con la cirugía con 
Tabla 3. Posibles complicaciones (seguridad)

\begin{tabular}{|c|c|c|}
\hline & \multicolumn{2}{|c|}{$\begin{array}{c}\text { Colecistectomía laparoscópica } \\
\text { habitual }\end{array}$} \\
\hline Sangrado leve $\left(<50 \mathrm{~cm}^{3}\right)$ & \multicolumn{2}{|c|}{$33(100 \%)$} \\
\hline Inestabilidad hemodinámica & \multicolumn{2}{|c|}{$0(0 \%)$} \\
\hline Uso de drenaje (Penrose) & \multicolumn{2}{|c|}{$14(42.42 \%)$} \\
\hline Tabla 4. Puntaje de la encu & esta de satisfaccic & \\
\hline Puntos & $\begin{array}{l}\text { Colecistectomía } \\
\text { laparoscópica } \\
\text { habitual }\end{array}$ & $\begin{array}{c}\text { Colecistectomía } \\
\text { laparoscópica } \\
\text { ambulatoria }\end{array}$ \\
\hline 10 & $9(27.27 \%)$ & $12(31.57 \%)$ \\
\hline 11 & $5(15.15 \%)$ & $7(18.42 \%)$ \\
\hline 12 & $8(24.24 \%)$ & $7(18.42 \%)$ \\
\hline 13 & $3(9.09 \%)$ & $4(10.52 \%)$ \\
\hline 14 & $2(6.06 \%)$ & $3(7.89 \%)$ \\
\hline 15 & $2(6.06 \%)$ & $1(2.63 \%)$ \\
\hline 16 & $3(9.09 \%)$ & $3(7.89 \%)$ \\
\hline 17 & $0(0 \%)$ & $1(2.63 \%)$ \\
\hline 18 & $1(3.03 \%)$ & $0(0 \%)$ \\
\hline Prueba de hipótesis y $p$ & $T=0.5372$ & $=0.5929)$ \\
\hline
\end{tabular}

hospitalización, y por tanto se considera que es igual de efectiva y segura.

El objetivo central de nuestro estudio fue evaluar el grado de satisfacción por la atención recibida, el cual fue ligeramente mayor en el grupo de CLA, aun sin ser estadísticamente significativo, pero consideramos clínicamente más valioso el hecho de que esté cambiando la idea de que es mejor estar en el hospital, al menos el primer día, para sentirse mejor cuidado; ahora, el paciente, confiando en la información de su médico, acepta bien la oportunidad de regresar a la comodidad de su hogar en esa etapa de recuperación inmediata.

Por todo lo anterior, la CLA puede considerarse un procedimiento de calidad al ser seguro, eficaz y altamente satisfactorio para los pacientes siempre que se seleccionen bien los casos, y siempre que la institución cuente con las instalaciones y el equipo acordes a la normatividad vigente, así como con el personal adecuado, es decir, con entrenamiento específico para el desarrollo de las habilidades y destrezas laparoscópicas, incluyendo, idealmente, la certificación por el Consejo de la especialidad correspondiente.

\section{Conclusiones}

La eficacia, la seguridad y la satisfacción de los pacientes sometido a CLA son semejantes a las de los pacientes tratados con CLH, con una buena selección de los casos para lograr un adecuado control del dolor posoperatorio, que es la principal objeción para la ambulatorización de la CL. Por otro lado, aunque se logra una recuperación semejante con ambos abordajes, con la CLA se obtiene una más pronta reinserción a la vida laboral sin incrementar la frecuencia de complicaciones posquirúrgicas.

Una ventaja inmediatamente perceptible de la CLA para los hospitales es una mayor disminución en los costos de atención y los insumos, pero también es una ventaja de este procedimiento que el flujo de pacientes en quirófano sin necesidad de ocupación de camas censables permite una mayor movilidad administrativa sin afectar la calidad de la atención.

Con respecto al uso de drenaje, el paciente tiene una similar recuperación sin su uso, por lo que se sugiere suspender su empleo sistemático ya que no ofrece ningún beneficio para el paciente y, al contrario, puede llegar a incrementar el riesgo de infección.

Mediante la encuesta aplicada se demuestra que es muy semejante la satisfacción de los pacientes tanto en el ámbito ambulatorio como en el de ingreso hospitalario, ya que ambos grupos sienten la misma confianza, tranquilidad y seguridad en su recuperación, pero en el grupo de CLA, más que solo evitar la estancia hospitalaria, la ventaja es que se puede volver el mismo día a la comodidad del hogar.

La CLA es tan efectiva y segura como la CLH, por lo que ya puede considerarse como el nuevo método de referencia para el manejo quirúrgico de la patología biliar no complicada, aceptada tanto por el médico como por el paciente, con un alto grado de satisfacción de este último, y que permite elevar la calidad en la atención por parte de la institución. 


\section{Conflicto de intereses}

Los autores declaran que no existen conflictos de intereses.

\section{Financiamiento}

Los autores declaran que no recibieron patrocinio para llevar a cabo esta investigación.

\section{Responsabilidades éticas}

Protección de personas y animales. Los autores declaran que los procedimientos seguidos se conformaron a las normas éticas del comité de experimentación humana responsable y de acuerdo con la Asociación Médica Mundial y la Declaración de Helsinki.

Confidencialidad de los datos. Los autores declaran que han seguido los protocolos de su centro de trabajo sobre la publicación de datos de pacientes.

Derecho a la privacidad y consentimiento informado. Los autores han obtenido el consentimiento informado de los pacientes y/o sujetos referidos en el artículo. Este documento obra en poder del autor de correspondencia.

\section{Bibliografía}

1. García CJ, Ramírez AFJ. Colecistectomía de urgencia laparoscópica versus abierta. Cir Gen. 2012;34:174-8.

2. Hernández IRS, Rivero SJL, Quezada Al, Castillo GR, Flores RJF, Ávila RJL. Colecistectomía laparoscópica ambulatoria en un hospital de segundo nivel de atención. Cir Gen. 2008;30:13-6.

3. Vega-Vega M, Orlich-Carranza C, Gómez-Hernández K. Colecistectomía laparoscópica ambulatoria. Reporte preliminar de 100 casos. Acta Med Costarricence. 2006;48:119-22.

4. Nazar JC, Zamora HM, González AA. Cirugía ambulatoria: selección de pacientes y procedimientos quirúrgicos. Rev Chil Cir. 2015;67:207-13.

5. Widjaja SP, Fischer H, Brunner AR, Honigmann P, Metzger J. Acceptance of ambulatory laparoscopic cholecystectomy in central Switzerland. World J Surg. 2017;41:2731-4.

6. Llorens-León R, Navarro-Bravo R, Garrido-Babío JM, Arias A, Aguirre-Jaime A. SATISCORE : un cuestionario para valorar la satisfacción del paciente tras cirugía cardiaca. Cir Cardiov. 2014;21:239-45.

7. Reglamento de la Ley General de Salud en Materia de Investigación para la Salud. Disponible en: http://www.salud.gob.mx/unidades/cdi/nom/ compi/rlgsmis.html

8. Declaración de Helsinki. Disponible en: https://www.wma.net/policies-post/wma-declaration-of-helsinki-ethical-principles-for-medical-research-involving-human-subjects

9. Justo JJM, Prado OE, Theurel VG, de la Rosa PR, Lozano EA. Colecistectomía laparoscópica ambulatoria. Una buena alternativa. Cir Gen. 2004;26:306-10.

10. López EG, Zavala GJF, Paipilla MO, Lee RS. Colecistectomías laparoscópicas realizadas en Unidad de Cirugía Ambulatoria. Cir Gen. 2011; 33:104-10. 